\title{
Improved Performance Using Nonlinear Components in Power Control Algorithms
}

\author{
Fredrik Gunnarsson, Fredrik Gustafsson and Jonas Blom \\ Department of Electrical Engineering \\ Linköpings universitet, SE-581 83 Linköping, Sweden \\ WWW: http://www. control.isy.liu.se \\ Email: fred@isy.liu.se, fredrik@isy.liu.se, \\ jb@isy.liu.se
}

April 16, 1999

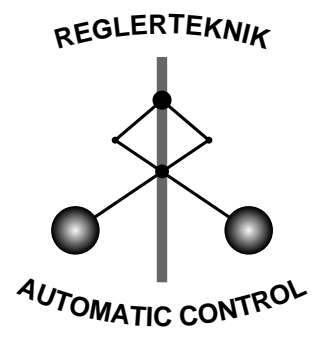

LINKÖPING

Report no.: LiTH-ISY-R-2148

Submitted to VTC'99, Houston, TX, USA

Technical reports from the Automatic Control group in Linkping are available by anonymous ftp at the address ftp.control.isy.liu.se. This report is contained in the compressed postscript file 2148.ps.Z. 



\begin{abstract}
When employing power control algorithms in real systems there are a number of problems that have to be considered, of which some are addressed in this work. It is probably impossible to solve the problems using a linear controller, and as an extension, various nonlinear components and their applicability are discussed. Problems due to hardware and external constraints have been identified in previous work, and here anti-reset windup techniques are reviewed and employed. Previous proposals can be seen as special cases. Sometimes it is desirable to employ several algorithms in parallel, and then use a device to state priorities, i.e., to switch between the algorithms. The applicability of switches and selectors are described and exemplified as proposed solutions to the low interference problem, where a $\mathrm{C} / \mathrm{I}$ balancing algorithm causes a user to use a too low a power. The benefits of using the proposed nonlinear components are further illustrated by network simulations.
\end{abstract}

Keywords: Cellular radio systems; Power Control Algorithms; Anti-reset windup; Selectors; Local loop design 


\section{Introduction}

For proper operation of a high-capacity cellular radio system, power control is an essential feature. Among others, there are three important aspects to consider.

- Extracting relevant information from the available measurements.

- Design a linear power control algorithm and tune the parameter for optimized performance.

- Incorporate nonlinear components to handle constraints and priorities.

The last issue is dealt with here, while the others are discussed in $[11,12]$.

With the objective of serving as many users as possible, it is important to utilize the available bandwidth efficiently. The main issue in this work is methods to assign appropriate transmission powers to the users with the intention of maximizing the capacity, while providing sufficient transmission quality to the users. Distributed power control algorithms have previously been studied in $[8,7,1,17,14,2,16,12]$. Most algorithms strive to balance the carrier-tointerference ratio $(\mathrm{C} / \mathrm{I})$. For each user, the strategy can be seen as a tradeoff between the user's desire to use maximum power in order to get best possible quality, and the system's desire to force the user to use less power in order to reduce the interference experienced by others.

These algorithms perform well in rather ideal cases, but in real systems there are a number of effects that hamper the performance. Firstly, measuring and control signaling takes time, which result in time delays in the system. The effects are studied in $[12,10]$. Secondly, the possible output powers of the transmitters are constrained due to physical limits and quantization. Furthermore, different external constraints such as the use of maximum power on specific channels affect the output power. This may lead to an oscillatory behavior of the algorithm [10]. Schemes that consider constraints are studied in $[2,9,3,6]$.

Thirdly, the low interference problem manifests itself when the load of the system is low, for example at night. When using a $\mathrm{C} / \mathrm{I}$ balancing algorithm, the power will be decreased to compensate for the low interference, resulting in an unnecessarily weak carrier signal, sensitive to disturbances and fluctuations.

Finally, when the system load is high, it may not be possible to support all users. A C/I balancing algorithm will result in increasing powers up to the upper output limit, without meeting the quality requirements. This party effect is discussed in $[1,18]$.

It is probably impossible to design a linear power control algorithm (most popular algorithms can be seen as linear in logarithmic scale [12]) that solves all the challenges listed above. In this work, the extension of incorporating nonlinear components in the algorithm is discussed. First we review some basic definitions and state the problems formally. Then the applicability of anti-reset windup, rate limiters and selectors are discussed followed by simulations and conclusions. 


\section{System Model}

All signal gains and power levels will be represented using logarithmic scales. Assume that the $m$ mobile stations on a specific radio channel are transmitting using the powers $p_{i}(t), i=1, \ldots, m$. The signal between mobile station $i$ and base station $j$ is attenuated by the signal gain $g_{i j}(t)(<0)$. Thus the corresponding connected base station will experience a desired carrier signal $C_{i}(t)=p_{i}(t)+g_{i i}(t)$ and an interference plus noise $I_{i}(t)$ (values in logarithmic scale). The carrier-to-interference ratio at base station $i$ is defined by

$$
\gamma_{i}(t)=p_{i}(t)+g_{i i}(t)-I_{i}(t) .
$$

The Quality of Service (QoS) is measured using either $\gamma_{i}(t)$ or average Frame Erasure Rate (FER). A user is considered satisfied when his average FER is below 0.02 [15], or alternly when his average $\mathrm{C} / \mathrm{I}$ is above a threshold $\gamma_{r e f}$. A system, where it is possible to assign powers to meet the requirements for all users is referred to as feasible [19].

Furthermore, it is assumed that a nominal linear power control algorithm is provided, designed to achieve acceptable quality when the system load is moderate. We will use the PI controller designed in [12], where time delays are considered in the design phase (reflected in the parameter values).

$$
\begin{aligned}
e(t) & =\left(\gamma_{r e f}-\gamma(t)\right) \\
x(t+1) & =x(t)+K_{i} T_{s} e(t) \\
p(t) & =K_{p} e(t)+x(t+1),
\end{aligned}
$$

where $x(t)$ is the integrator state, $K_{p}, K_{i}$ parameters, and $T_{s}$ the sampling interval (note that $K_{p}=0, K_{i} T_{s}=1$ corresponds to the DPC algorithm [8]).

If the computed output power is $p(t)$, we let $f(p(t))$ denote the true output of the transmitter. Thus the static nonlinearity $f(\cdot)$ describes the total effect of hardware and external constraints.

Time delays are expressed using the delay operator $q$, defined by

$$
q^{-n} p(t)=p(t-n) .
$$

Here power output and measurement delays of $n_{p}$ and $n_{m}$ samples respectively are discussed. From the view of a decentralized controller, the surrounding environment can be depicted as in Figure 1. The available information consists of measurement reports, from which an estimator extracts relevant information. Here it is assumed that at least the $\mathrm{C} / \mathrm{I}(\hat{\gamma})$, and the mean interference $\left(\hat{m}_{I}\right)$ are estimated. Such an estimator is described in [11].

\section{Nonlinear Components}

System nonlinearities are often classified as either inherent or intentional nonlinearities. The first category consist of components that we cannot affect in 


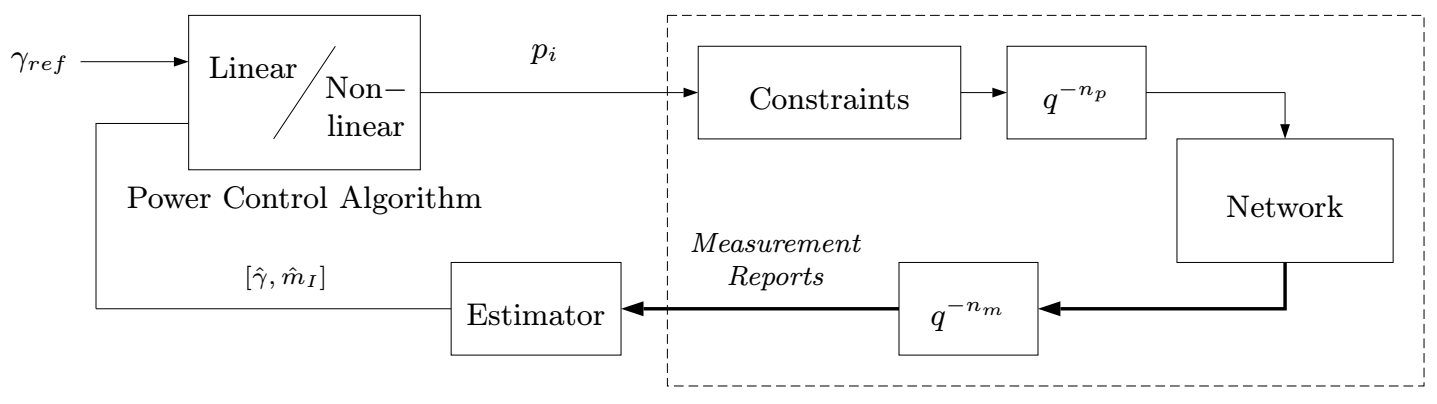

Environment

Figure 1: A schematic view of the surrounding Environment as seen by a decentralized controller. The Network block incorporates the effects caused by the radio channel, such as power gain, noise and interfering transmitters. The transmission powers are individually assigned using an algorithm comprising both linear and nonlinear components in order to adapt to different situations.

the design at the moment, such as output power constraints. On the other hand intentional nonlinearities are those which are deliberately introduced in the system. In this article the focus is on the latter, possibly introduced to reduce the effects of the former.

\subsection{Anti-Reset Windup}

Consider a case where the $\mathrm{C} / \mathrm{I}$ is well above $\gamma_{r e f}$ despite the fact that we are transmitting using minimal power. Since the error $e(t)$ is negative, the state $x(t)$ in (2) will continue to decrease. When the $\mathrm{C} / \mathrm{I}$ degrades and there is a need to increase the power, the low value (maybe negative) of the state reduces the controller's ability to increase the power fast.

In general, the problem is that the inner state of the dynamical controller is unaware of that the computed power is different from the true output power. This results in inner states "winding up" (or down). The problem is discussed for PID-controllers in [5], and the proposed solutions are referred to as anti-reset windup. Assume that the constraints can be described as a static nonlinear function $f(\cdot)$ (not necessarily analytical). An intuitive solution is to update the state again after computing the power level:

$$
x(t+1):=x(t+1)+\frac{T_{s}}{T_{t}}(f(p(t+1))-p(t+1)),
$$

where $T_{t}$ is the tracking parameter. If $T_{t}=T_{s}$, the state will exactly correspond to the actual output. Note that when $K_{p}=0, K_{i} T_{s}=1, T_{t}=T_{s}$ and $f(\cdot)$ is a saturation, we obtain the Distributed Constrained Power Control (DCPC) algorithm [9] as a special case.

A more complicated power control algorithm of higher order (e.g. as in [12]) needs special treatment. A general linear algorithm can be described in state 
space form as

$$
\begin{aligned}
x(t+1) & =A x(t)+B y(t) \\
p(t) & =C x(t)+D y(t),
\end{aligned}
$$

where $A(n \times n), B(n \times m), C(m \times n), D(m \times m)$ are matrices of given orders, $y(t)$ is a measurement vector, and $n$ is the dimension of the state vector. The order of the input vector $m$ is in a typical power control case equal to one (e.g. $\left.y(t)=e(t)=\gamma_{r e f}-\gamma(t)\right)$ or two (e.g. $y(t)=\left[\begin{array}{ll}\gamma_{r e f} & \gamma(t)\end{array}\right]^{T}$ ). An observer-based anti-windup scheme (see [13] for details) can be implemented as

$$
\begin{aligned}
x(t+1)= & A x(t)+B y(t)+ \\
& +L(f(p(t))-C x(t)-D y(t)) \\
p(t)= & C x(t)+D y(t),
\end{aligned}
$$

where the $(n \times m)$-matrix $L$ is a design parameter.

The static function $f(\cdot)$ should not only incorporate the inherent nonlinearities, but also all the intentional nonlinearities, such as those discussed in the rest of the section.

\subsection{Selector and Switch}

Sometimes it is desirable to employ several algorithms in parallel, and then use a device to state priorities, i.e. to switch between the algorithms. We will describe two possible solutions: the selector and the switch.

Consider the low interference problem, where a $\mathrm{C} / \mathrm{I}$ balancing algorithm computes a very low power to use when the interference is low. The system requirement that a user should use a low power in order not to disturb others does not hold, and instead the user requirement of using a high power to obtain good quality should be prioritized. This could be achieved by using a maximum selector as below

$$
p(t+1)=\max \left(p_{\mathrm{PCA}}(t+1), h\left(m_{I}(t)\right)\right),
$$

where $p_{\mathrm{PCA}}(t+1)$ is computed by a nominal power control algorithm and $h(\cdot)$ is a function of the measured or estimated (see e.g. $[6,11])$ average interference $\hat{m}_{I}$. This function may be constructed as in Figure 2 .

The device could also be implemented as a switch

$$
p(t+1)= \begin{cases}p_{\mathrm{PCA}}(t+1), & p_{\mathrm{PCA}}(t+1) \geq h\left(\hat{m}_{I}(t)\right) \\ h\left(\hat{m}_{I}(t)\right) & \text { otherwise }\end{cases}
$$

In other cases it may be interesting to switch between several dynamical controllers. For example, we might want to switch to the AAW-algorithm described in in [1] when the interference is high. Then it is important to apply anti-windup techniques to each of the controller, so that the internal states corresponds to the actual output, which possibly is computed using the other dynamical controller. 


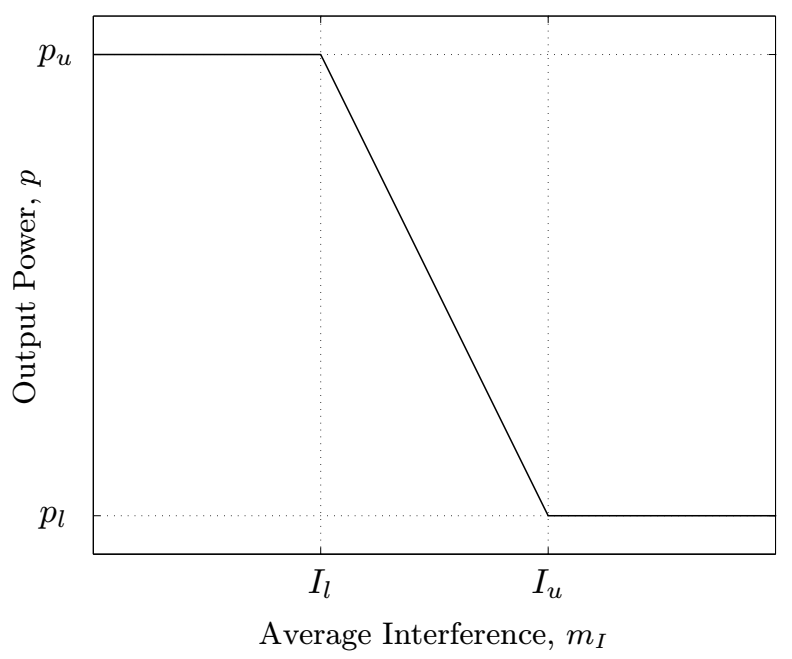

Figure 2: A possible choice of nonlinear function to use with the maximum selector. When the interference decreases the output power increases.

\subsection{Relay}

In some controllers, the output is an up/down command based whether the input is below or above a threshold. As an example, consider the inner loop power control of CDMA systems as discussed in [4], where the power is updated according to

$$
p(t+1)=p(t)+\Delta \operatorname{sign}\left(\gamma_{r e f}-\gamma(t)\right),
$$

where $\Delta$ is the step size in $\mathrm{dB}$. The sign function can schematically be represented as a relay. The main incentive for using the relay, is that bandwidth efficient binary command signaling can be used, which is very interesting when the update rate is high. Relays and their effects are further studied in [6].

\subsection{Rate Limiter}

When the C/I increases rapidly, possibly due to favorable propagation conditions, it may not be desired to decrease the power just as fast. The conditions may degrade rapidly later on, and therefore it may be appropriate to force the control algorithm to decrease the power more carefully. This is achieved by a rate limiter, which limits the rate of change downwards to $\delta \mathrm{dB}$. In order to avoid bad quality as much as possible, it is not recommended to use a similar limit for upward changes. As an example, the relay described previously implies a limited rate of change. 


\section{Simulations}

The nonlinear components are studied using a simulator, which represents a frequency hopping GSM system with the actual interfaces (measurements available in quantized reports [11]). It is further described in [6]. The simulator parameters are summarized in Table 1 . Focus on an individual, which has bad

\begin{tabular}{ll}
\hline Frequency band & $900 \mathrm{MHz}$ \\
Antennas & Sectorized \\
Cell radius & $1000 \mathrm{~m}$ \\
Cell layout & $5 \times 5$ clusters \\
& of 9 cells \\
Frequency hopping & Pseudo-random \\
Control sample interval & $T_{c}=0.48 \mathrm{~s}$ \\
Burst time & $0.577 \mathrm{~ms}$ \\
Mobile station & GSM class 4 \\
Mean mobile station speed & $50 \mathrm{~km} / \mathrm{h}$ \\
Power output delay & $n_{p}=1$ sample \\
Measurement delay & $n_{m}=1$ sample \\
\hline
\end{tabular}

Table 1: System simulation parameters.

quality when constant maximum powers is used in the system, and the intention is to study possible improvements when applying the different strategies. After about $14 \mathrm{~s}$, new mobile stations are establishing connections and therefore, the interference is increased abruptly as seen in Figure 3. By applying the Icontroller, the situation is improved, but instead problems are introduced at $4 \mathrm{~s}$, due to a too low a carrier. The problems are finally avoided by introducing the nonlinear components. The results using different strategies are summarized in Table 2.

The overall performance will for clarity be presented as improvements relative to using constant maximum powers. More specifically, we present the number of satisfied users when using different startegies relativ to the number of satisfied users when no control is employed. As seen in Figure 4, the I-controller performs bad when the load is low - even worse than not controlling the power at all. The problems are avoided by introducing the selector.

\section{Conclusions}

When employing power control algorithms in real systems there are a number of problems that have to be considered, of which some are addressed in this work. It is probably impossible to solve the problems using a linear controller, and as an extension, various nonlinear components and their applicability are discussed.

Due to hardware and external constraints, the power computed by a linear 
a.

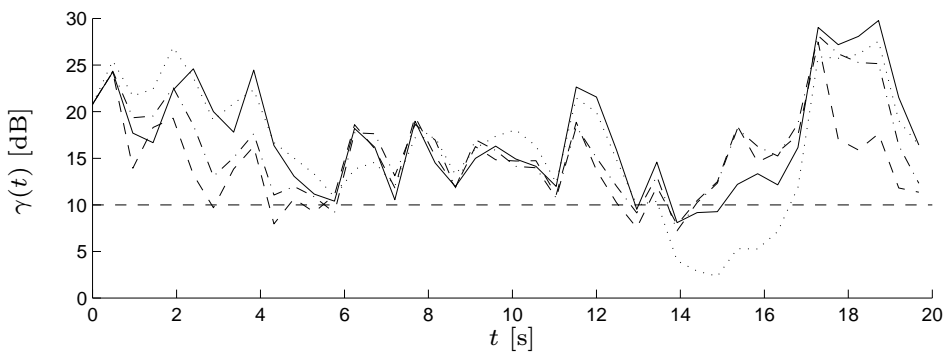

b.

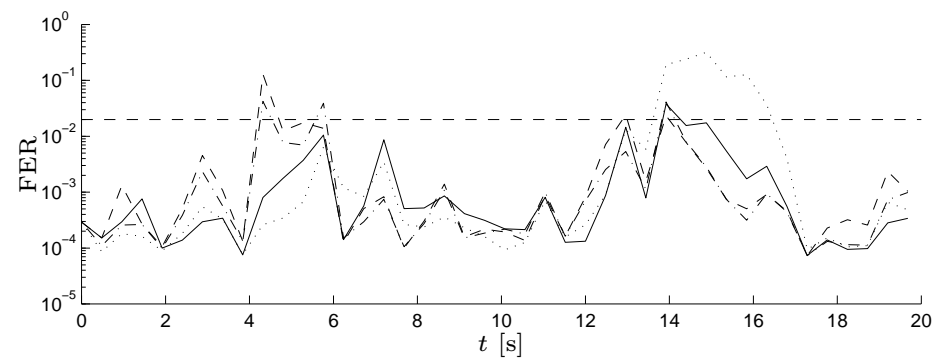

Figure 3: The perceived quality over time at a specific receiver given by a. $\mathrm{C} / \mathrm{I}$ and b. FER. The requirements are specified by the horizontal dashed line, and the algorithms considered are the I-controller (dashed), rate limiter (dashdotted) and selector (solid). For comparison, the dotted line corresponds to the case when maximum power is used.

power control and the actual output power at the transmitter may be different. This can result in an undesirable oscillatory behaviour or slow responses. For that matter, anti-reset windup techniques are reviewed and applied. Previous proposals can be seen as special cases.

Sometimes it is desirable to employ several algorithms in parallel, and then use a device to state priorities, i.e., to switch between the algorithms. The applicability of switches and selectors are described and exemplified as a proposed solutions to the low interference problem. In general, a C/I balancing algorithm can be seen as a tradeoff between the requirements from the user (high power required) and the system (low power desired). However, in case of a low system load, the user should be prioritized and allowed to use a high power to maximize the quality. The designed solution, a maximum selector, is employed and compared to using a rate limiter.

The benefits of using the proposed nonlinear components are further illustrated by network simulations. 


\begin{tabular}{|l||c|}
\hline Algorithm & Average FER \\
\hline \hline Constant power & 0.0255 \\
\hline I-controller & 0.0062 \\
\hline Rate limiter & 0.0041 \\
\hline Selector & 0.0031 \\
\hline
\end{tabular}

Table 2: Comparison of different control strategies in terms of satisfaction of an individual user. All the strategies result in quality that meets the requirement $\mathrm{E}[\mathrm{FER}] \leq 0.02$, but the I-controller introduces some new problems which are reduced when using the rate limiter and eliminated when using the selector, and therefore an even better quality is obtained.

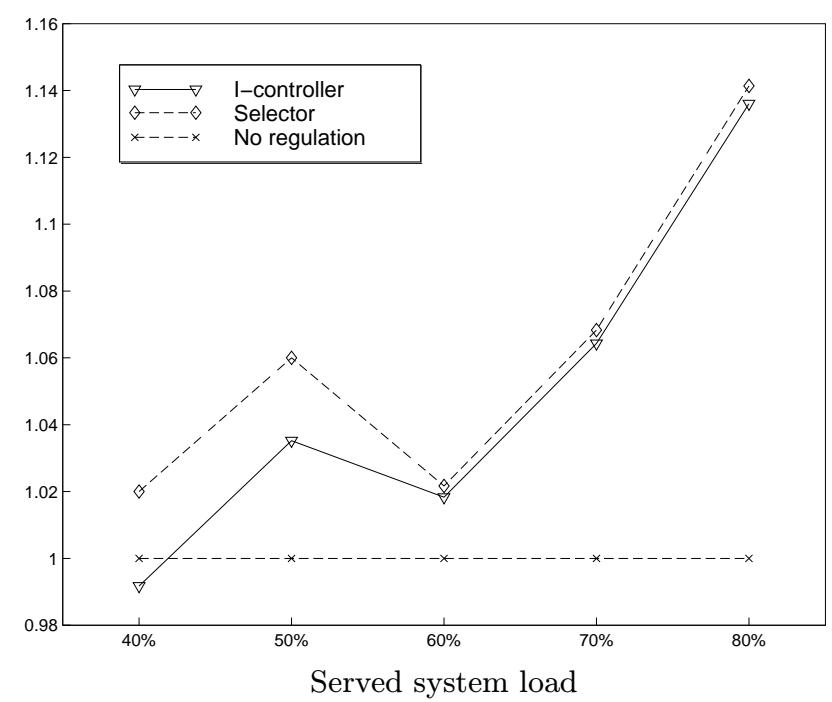

Figure 4: The percentage of satisfied customers for various served system load and algorithms relative to the number of satisfied customers when using constant maximum power. 


\section{References}

[1] M. Almgren, H. Andersson, and K. Wallstedt. Power control in a cellular system. In Proc. IEEE Vehicular Technology Conference, Stockholm, Sweden, June 1994.

[2] E. Anderlind. Resource Allocation in Multi-Service Wireless Access Networks. PhD thesis, Radio Comm. Systems Lab., Royal Inst. Technology, Stockholm, Sweden, October 1997.

[3] M. Andersin, Z. Rosberg, and J. Zander. Distributed discrete power control in cellular PCS. Wireless Personal Communications, 6(3), 1998.

[4] S. Ariyavisitakul. Signal and interference statistics of a CDMA system with feedback power control - part II. IEEE Transactions on Communications, 42(2), 1994.

[5] K. Åström and T. Hägglund. PID Controllers: Theory, Design, and Tuning. Instrument Society of America, Research Triangle Park, NC, USA, second edition, 1995.

[6] J. Blom and F. Gunnarsson. Power Control in Cellular Radio Systems. Licenciate Thesis, Linköpings universitet, Sweden, June 1998. Available at http://www. control.isy.liu.se.

[7] G. J. Foschini and Z. Miljanic. A simple distributed autonomus power control algorithm and its covergence. IEEE Transactions on Vehicular Technology, 42(4), 1993.

[8] S. A. Grandhi, R. Vijayan, and D. J. Goodman. Distributed power control in cellular radio systems. IEEE Transactions on Communications, 42(2), 1994.

[9] S. A. Grandhi, J. Zander, and R. Yates. Constrained power control. Wireless Personal Communications, 2(1), 1995.

[10] F. Gunnarsson, J. Blom, and F. Gustafsson. Power control in cellular systems subject to constraints and time delays. In Proc. IEEE Global Telecommunications Conference, Sydney, Australia, November 1998.

[11] F. Gunnarsson, J. Blom, and F. Gustafsson. Estimation of the carrierto-interference ratio in cellular radio systems. In Proc. IEEE Vehicular Technology Conference, Houston, TX, USA, May 1999.

[12] F. Gunnarsson, F. Gustafsson, and J. Blom. Pole placement design of power control algorithms. In Proc. IEEE Vehicular Technology Conference, Houston, TX, USA, May 1999.

[13] M. V. Kothare, P. J. Campo, M. Morari, and C. N. Nett. A unified framework for the study of anti-windup designs. Automatica, 30(12), 1994.

[14] T. H. Lee and J. C. Lin. A study on the distributed power control for cellular mobile systems. In Proc. IEEE Vehicular Technology Conference, Atlanta, GA, USA, April 1996. 
[15] H. Olofsson. Improved quality estimation for use in simulation of wireless TDMA systems. In Proc. IEEE International Conference on Universal Personal Communications, San Diego, CA, USA, October 1997.

[16] S. Ulukus and R. Yates. Stochastic power control for cellular radio systems. IEEE Transactions on Communications, 46(6), 1998.

[17] R. D. Yates. A framework for uplink power control in cellular radio systems. IEEE Journal on Selected Areas in Communications, 13(7), September 1995 .

[18] R. D. Yates, S. Gupta, C. Rose, and S. Sohn. Soft dropping power control. In Proc. IEEE Vehicular Technology Conference, Phoenix, AZ, USA, May 1997.

[19] J. Zander. Distributed cochannel interference control in cellular radio systems. IEEE Transactions on Vehicular Technology, 41(3), August 1992. 